\title{
Oscillatory behavior of third-order nonlinear neutral delay differential equations
}

Ying Jiang ${ }^{1}$, Cuimei Jiang ${ }^{1}$ and Tongxing $\mathrm{Li}^{2,3^{*}}$

${ }^{*}$ Correspondence:

litongx2007@163.com

${ }^{2}$ School of Informatics, Linyi

University, Linyi, Shandong 276005,

P.R. China

${ }^{3}$ LinDa Institute of Shandong

Provincial Key Laboratory of Network Based Intelligent

Computing, Linyi University, Linyi,

Shandong 276005, P.R. China

Full list of author information is

available at the end of the article

\begin{abstract}
We provide an oscillation criterion for a class of third-order nonlinear neutral delay differential equations by using the double generalized Riccati substitutions. Our theorem complements and improves previous results. Two illustrative examples are included.
\end{abstract}

MSC: $34 \mathrm{~K} 11$

Keywords: asymptotic behavior; oscillation; delay argument; third-order neutral differential equation

\section{Introduction}

This article is concerned with the oscillation and asymptotic behavior of a nonlinear thirdorder neutral delay differential equation

$$
\left(r(t)\left(z^{\prime \prime}(t)\right)^{\alpha}\right)^{\prime}+q(t) f(x(\sigma(t)))=0,
$$

where $t \geq t_{0}>0, z(t):=x(t)+p(t) x(\tau(t))$, and $\alpha \geq 1$ is a ratio of odd positive integers. We also suppose that the following assumptions hold:

$\left(A_{1}\right) r \in \mathrm{C}^{1}\left(\left[t_{0}, \infty\right),(0, \infty)\right), p, q \in \mathrm{C}\left(\left[t_{0}, \infty\right),[0, \infty)\right), \tau \in \mathrm{C}^{1}\left(\left[t_{0}, \infty\right), \mathbb{R}\right), \sigma \in \mathrm{C}\left(\left[t_{0}, \infty\right), \mathbb{R}\right)$, and $q$ is not identically zero for large $t$;

$\left(A_{2}\right) \int_{t_{0}}^{\infty} r^{-1 / \alpha}(t) \mathrm{d} t=\infty$ and $0 \leq p(t) \leq p_{0}<\infty$;

$\left(A_{3}\right) \tau(t) \leq t, \sigma(t) \leq t$, and $\lim _{t \rightarrow \infty} \tau(t)=\lim _{t \rightarrow \infty} \sigma(t)=\infty$;

$\left(A_{4}\right) f \in \mathrm{C}(\mathbb{R}, \mathbb{R})$ and there exists a positive constant $k$ such that $f(u) / u^{\alpha} \geq k$ for all $u \neq 0$;

$\left(A_{5}\right) \tau^{\prime}(t) \geq \tau_{0}>0$ and $\tau \circ \sigma=\sigma \circ \tau$.

By a solution to equation (1.1) we mean a function $x \in \mathrm{C}\left(\left[T_{x}, \infty\right), \mathbb{R}\right), T_{x} \geq t_{0}$, which has the property $r\left(z^{\prime \prime}\right)^{\alpha} \in \mathrm{C}^{1}\left(\left[T_{x}, \infty\right), \mathbb{R}\right)$ and satisfies $(1.1)$ on the interval $\left[T_{x}, \infty\right)$. We consider only those solutions to (1.1) which satisfy condition $\sup \{|x(t)|: t \geq T\}>0$ for all $T \geq T_{x}$ and assume that (1.1) possesses such solutions. A solution of (1.1) is called oscillatory if it has arbitrarily large zeros on $\left[T_{x}, \infty\right)$; otherwise, it is said to be nonoscillatory.

In recent years, the oscillation theory of functional differential equations has received much attention since it has a great number of applications in engineering and natural sciences. For some related contributions on the oscillatory behavior of various classes of functional differential equations, we refer the reader to [1-16] and the references cited therein. In the following, we provide some background details that motivated our study.

(c) 2016 Jiang et al. This article is distributed under the terms of the Creative Commons Attribution 4.0 International License (http://creativecommons.org/licenses/by/4.0/), which permits unrestricted use, distribution, and reproduction in any medium, provided you give appropriate credit to the original author(s) and the source, provide a link to the Creative Commons license, and indicate if changes were made. 
Baculíková et al. [6] and Li and Rogovchenko [11] established several oscillation theorems for a second-order neutral differential equation

$$
\left(r(t)\left|z^{\prime}(t)\right|^{\alpha-1} z^{\prime}(t)\right)^{\prime}+q(t) f(x(\sigma(t)))=0, \quad z:=x+p \cdot(x \circ \tau)
$$

under the assumptions that $\left(A_{1}\right)-\left(A_{5}\right)$ hold and $\alpha>0$ is a constant. For oscillation of thirdorder neutral differential equations, Baculíková and Džurina [1, 2], Candan [7], and Džurina et al. [9] considered the couple of third-order neutral differential equations

$$
\left(a(t)\left((x(t) \pm p(t) x(\delta(t)))^{\prime \prime}\right)^{\gamma}\right)^{\prime}+q(t) x^{\gamma}(\tau(t))=0
$$

in the case where

$$
0 \leq p(t) \leq p_{0}<1
$$

Baculíková and Džurina [5] studied a class of third-order neutral differential equations

$$
\left(a(t)\left((x(t)+p(t) x(\delta(t)))^{\prime}\right)^{\gamma}\right)^{\prime \prime}+q(t) x^{\gamma}(\tau(t))=0,
$$

whereas Baculíková and Džurina [4], Jiang and Li [10], Li and Rogovchenko [12], Li et al. [14], and Xing et al. [16] considered a third-order neutral differential equation

$$
\left(r(t)(x(t)+p(t) x(\tau(t)))^{\prime \prime}\right)^{\prime}+q(t) x(\sigma(t))=0 .
$$

In particular, using the comparison method, Xing et al. [16] obtained the following result for equation (1.3); see ([16], Corollary 2.8).

Theorem 1.1 Assume that conditions $\left(A_{1}\right)-\left(A_{3}\right)$ and $\left(A_{5}\right)$ are satisfied, and let $\sigma^{-1} \in$ $\mathrm{C}^{1}\left(\left[t_{0}, \infty\right), \mathbb{R}\right),\left(\sigma^{-1}(t)\right)^{\prime} \geq \sigma_{0}>0$, and $\sigma(t)<\tau(t) \leq t$. If

$$
r^{\prime} \geq 0
$$

and

$$
\liminf _{t \rightarrow \infty} \int_{\tau^{-1}(\sigma(t))}^{t} \frac{s^{2} \bar{Q}(s)}{r(s)} \mathrm{d} s>\frac{2\left(\tau_{0}+p_{0}\right)}{\sigma_{0} \tau_{0} \mathrm{e}}
$$

where $\bar{Q}(t):=\min \left\{q\left(\sigma^{-1}(t)\right), q\left(\sigma^{-1}(\tau(t))\right)\right\}, \tau^{-1}$ and $\sigma^{-1}$ denote the inverse functions of $\tau$ and $\sigma$, respectively, then every solution $x$ of (1.3) is either oscillatory or satisfies $\lim _{t \rightarrow \infty} x(t)=0$.

It should be noted that assumptions (1.2) and (1.4) are restrictive conditions in the study of oscillation of (1.1) and research in this paper was strongly motivated by the recent contributions of Li and Rogovchenko [11,12], Li et al. [14], and Xing et al. [16]. Our principal goal is to establish an oscillation criterion for a nonlinear third-order neutral delay differential equation (1.1) which can be applied in the case when $p_{0}>1$ as well and without requiring condition (1.4). In the sequel, we use the following notation:

$$
f_{+}(t):=\max \{0, f(t)\}, \quad Q(t):=\min \{q(t), q(\tau(t))\}, \quad R(t):=\max \{r(t), r(\tau(t))\},
$$


and all functional inequalities are tacitly assumed to hold for all $t$ large enough, unless mentioned otherwise.

\section{Lemmas}

Lemma 2.1 Assume that conditions $\left(A_{1}\right)-\left(A_{4}\right)$ hold and $x$ is a positive solution of (1.1). Then there are only the following two possible cases for $z$ :

(I) $z(t)>0, z^{\prime}(t)>0, z^{\prime \prime}(t)>0$, and $\left(r\left(z^{\prime \prime}\right)^{\alpha}\right)^{\prime}(t) \leq 0$;

(II) $z(t)>0, z^{\prime}(t)<0, z^{\prime \prime}(t)>0$, and $\left(r\left(z^{\prime \prime}\right)^{\alpha}\right)^{\prime}(t) \leq 0$,

where $t \geq T, T \geq t_{0}$ is sufficiently large.

Proof The proof is similar to that of Baculíková and Džurina ([1], Lemma 1), and thus is omitted.

Lemma 2.2 Assume that conditions $\left(A_{1}\right)-\left(A_{5}\right)$ are satisfied. Let $x$ be a positive solution of (1.1) and the corresponding $z$ satisfy case (II) in Lemma 2.1. If

$$
\int_{t_{0}}^{\infty} \xi\left(\frac{1}{R(\xi)} \int_{\xi}^{\infty} Q(s) \mathrm{d} s\right)^{1 / \alpha} \mathrm{d} \xi=\infty
$$

then $\lim _{t \rightarrow \infty} x(t)=\lim _{t \rightarrow \infty} z(t)=0$.

Proof Note that there exist three constants $c_{1}, c_{2}$, and $c_{3}$ such that $\lim _{t \rightarrow \infty} r(t)\left(z^{\prime \prime}(t)\right)^{\alpha}=$ $c_{1} \geq 0, \lim _{t \rightarrow \infty} z^{\prime}(t)=c_{2} \leq 0$, and $\lim _{t \rightarrow \infty} z(t)=c_{3} \geq 0$. A similar analysis to that in ([12], Theorem 15) leads to the conclusion that $\lim _{t \rightarrow \infty} x(t)=\lim _{t \rightarrow \infty} z(t)=0$.

\section{Main results}

Theorem 3.1 Assume that conditions $\left(A_{1}\right)-\left(A_{5}\right)$ and (2.1) are satisfied. If there exist two functions $\rho \in C^{1}\left(\left[t_{0}, \infty\right),(0, \infty)\right)$ and $\delta \in C^{1}\left(\left[t_{0}, \infty\right),[0, \infty)\right)$ such that

$$
\int_{t_{*}}^{\infty}\left[2^{1-\alpha} k \rho(t) Q(t)\left(\frac{\int_{t_{2}}^{\sigma(t)} \int_{t_{1}}^{s} r^{-1 / \alpha}(u) \mathrm{d} u \mathrm{~d} s}{\int_{t_{1}}^{t} r^{-1 / \alpha}(u) \mathrm{d} u}\right)^{\alpha}-G(t)\right] \mathrm{d} t=\infty
$$

for a sufficiently large $t_{1} \geq t_{0}$ and for some $t_{*}>t_{2}>t_{1}$, where

$$
\begin{aligned}
G(t):= & \rho(t)\left[\left((r(t) \delta(t))^{\prime}-r(t) \delta^{1+1 / \alpha}(t)\right)\right. \\
& \left.+\frac{p_{0}^{\alpha}}{\tau_{0}}\left((r(\tau(t)) \delta(\tau(t)))^{\prime}-r(\tau(t)) \tau^{\prime}(t) \delta^{1+1 / \alpha}(\tau(t))\right)\right] \\
& +\frac{\rho(t) r(t)}{(\alpha+1)^{\alpha+1}}\left(\frac{\rho_{+}^{\prime}(t)}{\rho(t)}+(\alpha+1) \delta^{1 / \alpha}(t)\right)^{\alpha+1} \\
& +\frac{p_{0}^{\alpha}}{\tau_{0}^{\alpha+1}} \frac{\rho(t) r(\tau(t))}{(\alpha+1)^{\alpha+1}}\left(\frac{\rho_{+}^{\prime}(t)}{\rho(t)}+(\alpha+1) \delta^{1 / \alpha}(\tau(t)) \tau^{\prime}(t)\right)^{\alpha+1},
\end{aligned}
$$

then every solution $x$ of (1.1) is either oscillatory or satisfies $\lim _{t \rightarrow \infty} x(t)=0$.

Proof Suppose that $x$ is a nonoscillatory solution of (1.1) which, without loss of generality, is eventually positive. Then there exists a $t_{1} \geq t_{0}$ such that $x(t)>0, x(\tau(t))>0$, and 
$x(\sigma(t))>0$ for $t \geq t_{1}$. By Lemma 2.1, we observe that $z$ satisfies either (I) or (II) for $t \geq T$, where $T \geq t_{1}$ is large enough. We consider each of the two cases separately.

Assume first that case (I) holds. By virtue of (1.1) and $\left(A_{4}\right)$,

$$
\left(r(t)\left(z^{\prime \prime}(t)\right)^{\alpha}\right)^{\prime}=-q(t) f(x(\sigma(t))) \leq-k q(t) x^{\alpha}(\sigma(t)) \leq 0 .
$$

It follows from $\left(r(\tau(t))\left(z^{\prime \prime}(\tau(t))\right)^{\alpha}\right)^{\prime}=\left(r\left(z^{\prime \prime}\right)^{\alpha}\right)^{\prime}(\tau(t)) \tau^{\prime}(t)$ that there exists a $t_{2} \geq T$ such that, for $t \geq t_{2}$,

$$
p_{0}^{\alpha} \frac{\left(r(\tau(t))\left(z^{\prime \prime}(\tau(t))\right)^{\alpha}\right)^{\prime}}{\tau^{\prime}(t)} \leq-p_{0}^{\alpha} k q(\tau(t)) x^{\alpha}(\sigma(\tau(t))) .
$$

Using the latter inequality and condition $\tau^{\prime}(t) \geq \tau_{0}>0$, we get, for $t \geq t_{2}$,

$$
\frac{p_{0}^{\alpha}}{\tau_{0}}\left(r(\tau(t))\left(z^{\prime \prime}(\tau(t))\right)^{\alpha}\right)^{\prime} \leq-p_{0}^{\alpha} k q(\tau(t)) x^{\alpha}(\sigma(\tau(t))) .
$$

Combining (3.3) and (3.4) and using the assumption $\sigma \circ \tau=\tau \circ \sigma$, we conclude that

$$
\begin{aligned}
& \left(r(t)\left(z^{\prime \prime}(t)\right)^{\alpha}\right)^{\prime}+\frac{p_{0}^{\alpha}}{\tau_{0}}\left(r(\tau(t))\left(z^{\prime \prime}(\tau(t))\right)^{\alpha}\right)^{\prime} \\
& \quad \leq-k\left(q(t) x^{\alpha}(\sigma(t))+p_{0}^{\alpha} q(\tau(t)) x^{\alpha}(\sigma(\tau(t)))\right) \\
& \quad \leq-k \min \{q(t), q(\tau(t))\}\left(x^{\alpha}(\sigma(t))+p_{0}^{\alpha} x^{\alpha}(\tau(\sigma(t)))\right) \\
& \quad=-k Q(t)\left(x^{\alpha}(\sigma(t))+p_{0}^{\alpha} x^{\alpha}(\tau(\sigma(t)))\right) .
\end{aligned}
$$

Using condition $0 \leq p(t) \leq p_{0}<\infty$ and the inequality (see ([3], Lemma 1))

$$
x_{1}^{\alpha}+x_{2}^{\alpha} \geq \frac{1}{2^{\alpha-1}}\left(x_{1}+x_{2}\right)^{\alpha},
$$

where $\alpha \geq 1, x_{1} \geq 0$, and $x_{2} \geq 0$, we have

$$
x^{\alpha}(\sigma(t))+p_{0}^{\alpha} x^{\alpha}(\tau(\sigma(t))) \geq \frac{\left(x(\sigma(t))+p_{0} x(\tau(\sigma(t)))\right)^{\alpha}}{2^{\alpha-1}} \geq \frac{z^{\alpha}(\sigma(t))}{2^{\alpha-1}} .
$$

Substitution of (3.6) into (3.5) implies that, for $t \geq t_{2}$,

$$
\left(r(t)\left(z^{\prime \prime}(t)\right)^{\alpha}\right)^{\prime}+\frac{p_{0}^{\alpha}}{\tau_{0}}\left(r(\tau(t))\left(z^{\prime \prime}(\tau(t))\right)^{\alpha}\right)^{\prime} \leq-\frac{k}{2^{\alpha-1}} Q(t)(z(\sigma(t)))^{\alpha} .
$$

For $t \geq t_{2}$, define a function $\omega$ by

$$
\omega(t):=\rho(t)\left[\frac{r(t)\left(z^{\prime \prime}(t)\right)^{\alpha}}{\left(z^{\prime}(t)\right)^{\alpha}}+r(t) \delta(t)\right] .
$$

Then $\omega(t)>0$ for $t \geq t_{2}$. Differentiation of (3.8) yields

$$
\begin{aligned}
\omega^{\prime}(t) & =\rho^{\prime}(t)\left[\frac{r(t)\left(z^{\prime \prime}(t)\right)^{\alpha}}{\left(z^{\prime}(t)\right)^{\alpha}}+r(t) \delta(t)\right]+\rho(t)\left[\frac{r(t)\left(z^{\prime \prime}(t)\right)^{\alpha}}{\left(z^{\prime}(t)\right)^{\alpha}}+r(t) \delta(t)\right]^{\prime} \\
& =\frac{\rho^{\prime}(t)}{\rho(t)} \omega(t)+\rho(t)(r(t) \delta(t))^{\prime}+\rho(t) \frac{\left(r(t)\left(z^{\prime \prime}(t)\right)^{\alpha}\right)^{\prime}}{\left(z^{\prime}(t)\right)^{\alpha}}-\alpha \rho(t) \frac{r(t)\left(z^{\prime \prime}(t)\right)^{\alpha+1}}{\left(z^{\prime}(t)\right)^{\alpha+1}} .
\end{aligned}
$$


By virtue of (3.8),

$$
\left(\frac{z^{\prime \prime}(t)}{z^{\prime}(t)}\right)^{\alpha+1}=\left(\frac{\omega(t)}{\rho(t) r(t)}-\delta(t)\right)^{(\alpha+1) / \alpha}
$$

Substituting (3.10) into (3.9), we conclude that

$$
\begin{aligned}
\omega^{\prime}(t)= & \frac{\rho^{\prime}(t)}{\rho(t)} \omega(t)+\rho(t)(r(t) \delta(t))^{\prime}+\rho(t) \frac{\left(r(t)\left(z^{\prime \prime}(t)\right)^{\alpha}\right)^{\prime}}{\left(z^{\prime}(t)\right)^{\alpha}} \\
& -\alpha \rho(t) r(t)\left(\frac{\omega(t)}{\rho(t) r(t)}-\delta(t)\right)^{(\alpha+1) / \alpha} \\
\leq & \frac{\rho_{+}^{\prime}(t)}{\rho(t)} \omega(t)+\rho(t)(r(t) \delta(t))^{\prime}+\rho(t) \frac{\left(r(t)\left(z^{\prime \prime}(t)\right)^{\alpha}\right)^{\prime}}{\left(z^{\prime}(t)\right)^{\alpha}} \\
& -\frac{\alpha}{(\rho(t) r(t))^{1 / \alpha}}(\omega(t)-\rho(t) r(t) \delta(t))^{1+1 / \alpha}
\end{aligned}
$$

Let

$$
A:=\omega(t) \quad \text { and } \quad B:=\rho(t) r(t) \delta(t) .
$$

Using the inequality (see ([13], Lemma 1 ) and note that $\alpha \geq 1$ is a ratio of odd integers)

$$
A^{1+1 / \alpha}-(A-B)^{1+1 / \alpha} \leq B^{1 / \alpha}\left[\left(1+\frac{1}{\alpha}\right) A-\frac{1}{\alpha} B\right], \quad A B \geq 0,
$$

we have

$$
\begin{aligned}
(\omega(t)-\rho(t) r(t) \delta(t))^{1+1 / \alpha} \geq & \omega^{1+1 / \alpha}(t)+\frac{1}{\alpha}(\rho(t) r(t) \delta(t))^{1+1 / \alpha} \\
& -\left(1+\frac{1}{\alpha}\right) \omega(t)(\rho(t) r(t) \delta(t))^{1 / \alpha} .
\end{aligned}
$$

Combining (3.11) and (3.13), we get

$$
\begin{aligned}
\omega^{\prime}(t) \leq & \frac{\rho_{+}^{\prime}(t)}{\rho(t)} \omega(t)+\rho(t)(r(t) \delta(t))^{\prime}+\rho(t) \frac{\left(r(t)\left(z^{\prime \prime}(t)\right)^{\alpha}\right)^{\prime}}{\left(z^{\prime}(t)\right)^{\alpha}}-\frac{\alpha}{(\rho(t) r(t))^{1 / \alpha}} \\
& \times\left[\omega^{(\alpha+1) / \alpha}(t)+\frac{1}{\alpha}(\rho(t) r(t) \delta(t))^{(\alpha+1) / \alpha}-\left(1+\frac{1}{\alpha}\right) \omega(t)(\rho(t) r(t) \delta(t))^{1 / \alpha}\right] \\
= & \rho(t) \frac{\left(r(t)\left(z^{\prime \prime}(t)\right)^{\alpha}\right)^{\prime}}{\left(z^{\prime}(t)\right)^{\alpha}}+\rho(t)(r(t) \delta(t))^{\prime}-\rho(t) r(t) \delta^{(\alpha+1) / \alpha}(t) \\
& +\left(\frac{\rho_{+}^{\prime}(t)}{\rho(t)}+(\alpha+1) \delta^{1 / \alpha}(t)\right) \omega(t)-\frac{\alpha}{(\rho(t) r(t))^{1 / \alpha}} \omega^{(\alpha+1) / \alpha}(t) .
\end{aligned}
$$

Let

$$
C:=\frac{\rho_{+}^{\prime}(t)}{\rho(t)}+(\alpha+1) \delta^{1 / \alpha}(t), \quad D:=\frac{\alpha}{(\rho(t) r(t))^{1 / \alpha}}, \quad \text { and } \quad u:=\omega(t)
$$


Using the inequality (see [11])

$$
C u-D u^{(\alpha+1) / \alpha} \leq \frac{\alpha^{\alpha}}{(\alpha+1)^{\alpha+1}} \frac{C^{\alpha+1}}{D^{\alpha}}, \quad D>0,
$$

we deduce from (3.14) that

$$
\begin{aligned}
\omega^{\prime}(t) \leq & \rho(t) \frac{\left(r(t)\left(z^{\prime \prime}(t)\right)^{\alpha}\right)^{\prime}}{\left(z^{\prime}(t)\right)^{\alpha}}+\rho(t)(r(t) \delta(t))^{\prime}-\rho(t) r(t) \delta^{1+1 / \alpha}(t) \\
& +\frac{\rho(t) r(t)}{(\alpha+1)^{\alpha+1}}\left(\frac{\rho_{+}^{\prime}(t)}{\rho(t)}+(\alpha+1) \delta^{1 / \alpha}(t)\right)^{\alpha+1} .
\end{aligned}
$$

Define another function $v$ by

$$
\nu(t):=\rho(t)\left[\frac{r(\tau(t))\left(z^{\prime \prime}(\tau(t))\right)^{\alpha}}{\left(z^{\prime}(\tau(t))\right)^{\alpha}}+r(\tau(t)) \delta(\tau(t))\right] .
$$

Then $v(t)>0$ for $t \geq t_{2}$. Differentiation of (3.17) implies that

$$
\begin{aligned}
v^{\prime}(t)= & \rho^{\prime}(t)\left[\frac{r(\tau(t))\left(z^{\prime \prime}(\tau(t))\right)^{\alpha}}{\left(z^{\prime}(\tau(t))\right)^{\alpha}}+r(\tau(t)) \delta(\tau(t))\right] \\
& +\rho(t)\left[\frac{r(\tau(t))\left(z^{\prime \prime}(\tau(t))\right)^{\alpha}}{\left(z^{\prime}(\tau(t))\right)^{\alpha}}+r(\tau(t)) \delta(\tau(t))\right]^{\prime} \\
= & \frac{\rho^{\prime}(t)}{\rho(t)} v(t)+\rho(t)(r(\tau(t)) \delta(\tau(t)))^{\prime}+\rho(t) \frac{\left(r(\tau(t))\left(z^{\prime \prime}(\tau(t))\right)^{\alpha}\right)^{\prime}}{\left(z^{\prime}(\tau(t))\right)^{\alpha}} \\
& -\alpha \rho(t) \frac{r(\tau(t))\left(z^{\prime \prime}(\tau(t))\right)^{\alpha+1} \tau^{\prime}(t)}{\left(z^{\prime}(\tau(t))\right)^{\alpha+1}} \\
= & \frac{\rho^{\prime}(t)}{\rho(t)} v(t)+\rho(t)(r(\tau(t)) \delta(\tau(t)))^{\prime}+\rho(t) \frac{\left(r(\tau(t))\left(z^{\prime \prime}(\tau(t))\right)^{\alpha}\right)^{\prime}}{\left(z^{\prime}(\tau(t))\right)^{\alpha}} \\
& -\alpha \rho(t) r(\tau(t)) \tau^{\prime}(t)\left(\frac{v(t)}{\rho(t) r(\tau(t))}-\delta(\tau(t))\right)^{(\alpha+1) / \alpha} \\
\leq & \frac{\rho_{+}^{\prime}(t)}{\rho(t)} v(t)+\rho(t)(r(\tau(t)) \delta(\tau(t)))^{\prime}+\rho(t) \frac{\left(r(\tau(t))\left(z^{\prime \prime}(\tau(t))\right)^{\alpha}\right)^{\prime}}{\left(z^{\prime}(\tau(t))\right)^{\alpha}} \\
& -\frac{\alpha \tau^{\prime}(t)}{(\rho(t) r(\tau(t)))^{1 / \alpha}(v(t)-\rho(t) r(\tau(t)) \delta(\tau(t)))^{1+1 / \alpha} .}
\end{aligned}
$$

Let

$$
A:=\nu(t) \quad \text { and } \quad B:=\rho(t) r(\tau(t)) \delta(\tau(t)) .
$$

Using inequality (3.12), we obtain

$$
\begin{aligned}
(v(t)-\rho(t) r(\tau(t)) \delta(\tau(t)))^{1+1 / \alpha} \geq & v^{1+1 / \alpha}(t)+\frac{1}{\alpha}(\rho(t) r(\tau(t)) \delta(\tau(t)))^{1+1 / \alpha} \\
& -\left(1+\frac{1}{\alpha}\right) v(t)(\rho(t) r(\tau(t)) \delta(\tau(t)))^{1 / \alpha} .
\end{aligned}
$$


Substituting (3.19) into (3.18), we get

$$
\begin{aligned}
v^{\prime}(t) \leq & \frac{\rho_{+}^{\prime}(t)}{\rho(t)} v(t)+\rho(t)(r(\tau(t)) \delta(\tau(t)))^{\prime}+\rho(t) \frac{\left(r(\tau(t))\left(z^{\prime \prime}(\tau(t))\right)^{\alpha}\right)^{\prime}}{\left(z^{\prime}(\tau(t))\right)^{\alpha}}-\frac{\alpha \tau^{\prime}(t)}{(\rho(t) r(\tau(t)))^{1 / \alpha}} \\
& \times\left[v^{1+1 / \alpha}(t)+\frac{1}{\alpha}(\rho(t) r(\tau(t)) \delta(\tau(t)))^{1+1 / \alpha}\right. \\
& \left.-\left(1+\frac{1}{\alpha}\right) v(t)(\rho(t) r(\tau(t)) \delta(\tau(t)))^{1 / \alpha}\right] \\
\leq & \rho(t) \frac{\left(r(\tau(t))\left(z^{\prime \prime}(\tau(t))\right)^{\alpha}\right)^{\prime}}{\left(z^{\prime}(\tau(t))\right)^{\alpha}}+\rho(t)(r(\tau(t)) \delta(\tau(t)))^{\prime}-\rho(t) r(\tau(t)) \delta^{1+1 / \alpha}(\tau(t)) \tau^{\prime}(t) \\
& +\left(\frac{\rho_{+}^{\prime}(t)}{\rho(t)}+(\alpha+1) \delta^{1 / \alpha}(\tau(t)) \tau^{\prime}(t)\right) v(t)-\frac{\alpha \tau^{\prime}(t)}{(\rho(t) r(\tau(t)))^{1 / \alpha}} v^{(\alpha+1) / \alpha}(t) .
\end{aligned}
$$

Let

$$
C:=\frac{\rho_{+}^{\prime}(t)}{\rho(t)}+(\alpha+1) \delta^{1 / \alpha}(\tau(t)) \tau^{\prime}(t), \quad D:=\frac{\alpha \tau^{\prime}(t)}{(\rho(t) r(\tau(t)))^{1 / \alpha}}, \quad \text { and } \quad u:=v(t)
$$

Using inequality (3.15), we deduce from (3.20) that

$$
\begin{aligned}
v^{\prime}(t) \leq & \rho(t) \frac{\left(r(\tau(t))\left(z^{\prime \prime}(\tau(t))\right)^{\alpha}\right)^{\prime}}{\left(z^{\prime}(\tau(t))\right)^{\alpha}}+\rho(t)(r(\tau(t)) \delta(\tau(t)))^{\prime}-\rho(t) r(\tau(t)) \tau^{\prime}(t) \delta^{1+1 / \alpha}(\tau(t)) \\
& +\frac{\rho(t) r(\tau(t))}{(\alpha+1)^{\alpha+1}\left(\tau^{\prime}(t)\right)^{\alpha}}\left(\frac{\rho_{+}^{\prime}(t)}{\rho(t)}+(\alpha+1) \delta^{1 / \alpha}(\tau(t)) \tau^{\prime}(t)\right)^{\alpha+1} .
\end{aligned}
$$

Since $z^{\prime \prime}(t)>0$ and $\tau(t) \leq t$, we have $z^{\prime}(\tau(t)) \leq z^{\prime}(t)$. Inequality (3.21) yields

$$
\begin{aligned}
v^{\prime}(t) \leq & \rho(t) \frac{\left(r(\tau(t))\left(z^{\prime \prime}(\tau(t))\right)^{\alpha}\right)^{\prime}}{\left(z^{\prime}(t)\right)^{\alpha}}+\rho(t)(r(\tau(t)) \delta(\tau(t)))^{\prime}-\rho(t) r(\tau(t)) \tau^{\prime}(t) \delta^{1+1 / \alpha}(\tau(t)) \\
& +\frac{\rho(t) r(\tau(t))}{(\alpha+1)^{\alpha+1}\left(\tau^{\prime}(t)\right)^{\alpha}}\left(\frac{\rho_{+}^{\prime}(t)}{\rho(t)}+(\alpha+1) \delta^{1 / \alpha}(\tau(t)) \tau^{\prime}(t)\right)^{\alpha+1} .
\end{aligned}
$$

Combining (3.16) and (3.22) and utilizing (3.7), we obtain

$$
\begin{aligned}
\omega^{\prime}(t)+\frac{p_{0}^{\alpha}}{\tau_{0}} v^{\prime}(t) \leq & \rho(t) \frac{\left(r(t)\left(z^{\prime \prime}(t)\right)^{\alpha}\right)^{\prime}+p_{0}^{\alpha} / \tau_{0}\left(r(\tau(t))\left(z^{\prime \prime}(\tau(t))\right)^{\alpha}\right)^{\prime}}{\left(z^{\prime}(t)\right)^{\alpha}} \\
& +\rho(t)\left((r(t) \delta(t))^{\prime}-r(t) \delta^{1+1 / \alpha}(t)\right) \\
& +\frac{\rho(t) r(t)}{(\alpha+1)^{\alpha+1}}\left(\frac{\rho_{+}^{\prime}(t)}{\rho(t)}+(\alpha+1) \delta^{1 / \alpha}(t)\right)^{\alpha+1} \\
& +\frac{p_{0}^{\alpha}}{\tau_{0}} \rho(t)\left((r(\tau(t)) \delta(\tau(t)))^{\prime}-r(\tau(t)) \tau^{\prime}(t) \delta^{1+1 / \alpha}(\tau(t))\right) \\
& +\frac{p_{0}^{\alpha}}{\tau_{0}} \frac{\rho(t) r(\tau(t))}{(\alpha+1)^{\alpha+1}\left(\tau^{\prime}(t)\right)^{\alpha}}\left(\frac{\rho_{+}^{\prime}(t)}{\rho(t)}+(\alpha+1) \delta^{1 / \alpha}(\tau(t)) \tau^{\prime}(t)\right)^{\alpha+1} \\
\leq & -2^{1-\alpha} k \rho(t) Q(t) \frac{(z(\sigma(t)))^{\alpha}}{\left(z^{\prime}(t)\right)^{\alpha}}+\rho(t)\left((r(t) \delta(t))^{\prime}-r(t) \delta^{1+1 / \alpha}(t)\right) \\
& +\frac{p_{0}^{\alpha}}{\tau_{0}} \rho(t)\left((r(\tau(t)) \delta(\tau(t)))^{\prime}-r(\tau(t)) \tau^{\prime}(t) \delta^{1+1 / \alpha}(\tau(t))\right)
\end{aligned}
$$




$$
\begin{aligned}
& +\frac{\rho(t) r(t)}{(\alpha+1)^{\alpha+1}}\left(\frac{\rho_{+}^{\prime}(t)}{\rho(t)}+(\alpha+1) \delta^{1 / \alpha}(t)\right)^{\alpha+1} \\
& +\frac{p_{0}^{\alpha}}{\tau_{0}^{\alpha+1}} \frac{\rho(t) r(\tau(t))}{(\alpha+1)^{\alpha+1}}\left(\frac{\rho_{+}^{\prime}(t)}{\rho(t)}+(\alpha+1) \delta^{1 / \alpha}(\tau(t)) \tau^{\prime}(t)\right)^{\alpha+1} \\
& =-2^{1-\alpha} k \rho(t) Q(t) \frac{(z(\sigma(t)))^{\alpha}}{\left(z^{\prime}(t)\right)^{\alpha}}+G(t),
\end{aligned}
$$

where $G$ is defined as in (3.2). By virtue of $\left(r(t)\left(z^{\prime \prime}(t)\right)^{\alpha}\right)^{\prime} \leq 0$, we have

$$
\begin{aligned}
z^{\prime}(t) & =z^{\prime}\left(t_{2}\right)+\int_{t_{2}}^{t} z^{\prime \prime}(s) \mathrm{d} s \\
& =z^{\prime}\left(t_{2}\right)+\int_{t_{2}}^{t} \frac{\left(r(s)\left(z^{\prime \prime}(s)\right)^{\alpha}\right)^{1 / \alpha}}{r^{1 / \alpha}(s)} \mathrm{d} s \\
& \geq r^{1 / \alpha}(t) z^{\prime \prime}(t) \int_{t_{2}}^{t} r^{-1 / \alpha}(s) \mathrm{d} s
\end{aligned}
$$

That is,

$$
z^{\prime}(t) r^{-1 / \alpha}(t)-z^{\prime \prime}(t) \int_{t_{2}}^{t} r^{-1 / \alpha}(s) \mathrm{d} s \geq 0
$$

which yields

$$
\left(\frac{z^{\prime}(t)}{\int_{t_{2}}^{t} r^{-1 / \alpha}(s) \mathrm{d} s}\right)^{\prime} \leq 0 .
$$

It follows from $\sigma(t) \leq t$ that

$$
\frac{z^{\prime}(\sigma(t))}{\int_{t_{2}}^{\sigma(t)} r^{-1 / \alpha}(s) \mathrm{d} s} \geq \frac{z^{\prime}(t)}{\int_{t_{2}}^{t} r^{-1 / \alpha}(s) \mathrm{d} s},
$$

and so

$$
\frac{z^{\prime}(\sigma(t))}{z^{\prime}(t)} \geq \frac{\int_{t_{2}}^{\sigma(t)} r^{-1 / \alpha}(s) \mathrm{d} s}{\int_{t_{2}}^{t} r^{-1 / \alpha}(s) \mathrm{d} s} .
$$

Using (3.25), we obtain

$$
\begin{aligned}
z(t) & =z\left(t_{3}\right)+\int_{t_{3}}^{t} z^{\prime}(s) \mathrm{d} s \\
& =z\left(t_{3}\right)+\int_{t_{3}}^{t} \frac{z^{\prime}(s)}{\int_{t_{2}}^{s} r^{-1 / \alpha}(u) \mathrm{d} u} \int_{t_{2}}^{s} r^{-1 / \alpha}(u) \mathrm{d} u \mathrm{~d} s \\
& \geq \frac{z^{\prime}(t)}{\int_{t_{2}}^{t} r^{-1 / \alpha}(u) \mathrm{d} u} \int_{t_{3}}^{t} \int_{t_{2}}^{s} r^{-1 / \alpha}(u) \mathrm{d} u \mathrm{~d} s
\end{aligned}
$$

for $t \geq t_{3}>t_{2}$, which implies that

$$
\frac{z(t)}{z^{\prime}(t)} \geq \frac{\int_{t_{3}}^{t} \int_{t_{2}}^{s} r^{-1 / \alpha}(u) \mathrm{d} u \mathrm{~d} s}{\int_{t_{2}}^{t} r^{-1 / \alpha}(u) \mathrm{d} u} .
$$


It follows now from (3.26) and (3.27) that

$$
\frac{(z(\sigma(t)))^{\alpha}}{\left(z^{\prime}(t)\right)^{\alpha}}=\left(\frac{z^{\prime}(\sigma(t))}{z^{\prime}(t)} \frac{z(\sigma(t))}{z^{\prime}(\sigma(t))}\right)^{\alpha} \geq\left(\frac{\int_{t_{3}}^{\sigma(t)} \int_{t_{2}}^{s} r^{-1 / \alpha}(u) \mathrm{d} u \mathrm{~d} s}{\int_{t_{2}}^{t} r^{-1 / \alpha}(u) \mathrm{d} u}\right)^{\alpha} .
$$

Substitution of (3.28) into (3.23) yields

$$
\omega^{\prime}(t)+\frac{p_{0}^{\alpha}}{\tau_{0}} v^{\prime}(t) \leq-2^{1-\alpha} k \rho(t) Q(t)\left(\frac{\int_{t_{3}}^{\sigma(t)} \int_{t_{2}}^{s} r^{-1 / \alpha}(u) \mathrm{d} u \mathrm{~d} s}{\int_{t_{2}}^{t} r^{-1 / \alpha}(u) \mathrm{d} u}\right)^{\alpha}+G(t) .
$$

Integrating (3.29) from $t_{4}\left(t_{4}>t_{3}\right)$ to $t$, we have

$$
\int_{t_{4}}^{t}\left[2^{1-\alpha} k \rho(v) Q(v)\left(\frac{\int_{t_{3}}^{\sigma(v)} \int_{t_{2}}^{s} r^{-1 / \alpha}(u) \mathrm{d} u \mathrm{~d} s}{\int_{t_{2}}^{v} r^{-1 / \alpha}(u) \mathrm{d} u}\right)^{\alpha}-G(v)\right] \mathrm{d} v \leq \omega\left(t_{4}\right)+\frac{p_{0}^{\alpha}}{\tau_{0}} v\left(t_{4}\right),
$$

which contradicts (3.1).

Assume now that case (II) holds. By virtue of Lemma $2.2, \lim _{t \rightarrow \infty} x(t)=0$. This completes the proof.

Remark 3.1 With an appropriate choice of the functions $\rho$ and $\delta$, one can derive from Theorem 3.1 a number of oscillation criteria for equation (1.1). The details are left to the reader.

\section{Examples and discussion}

We give the following examples to illustrate applications of Theorem 3.1.

Example 4.1 For $t \geq 1$, consider a third-order neutral delay differential equation

$$
\left(x(t)+2 x\left(\frac{t}{2}\right)\right)^{\prime \prime \prime}+\frac{\gamma}{t^{3}} x\left(\frac{t}{3}\right)=0,
$$

where $\gamma>0$ is a constant. Let $\alpha=k=1, r(t)=1, p(t)=p_{0}=2, \tau(t)=t / 2, \tau_{0}=1 / 2, q(t)=$ $\gamma / t^{3}, f(u)=u, \sigma(t)=t / 3, \sigma_{0}=3, \rho(t)=t$, and $\delta(t)=0$. It is not difficult to verify that conditions $\left(A_{1}\right)-\left(A_{5}\right)$ are satisfied and $G(t)=9 /(4 t)$. Noticing that $R(t)=\max \{r(t), r(\tau(t))\}=1$ and $Q(t)=\min \{q(t), q(\tau(t))\}=\min \left\{\gamma / t^{3}, 8 \gamma / t^{3}\right\}=\gamma / t^{3}$, we have

$$
\int_{t_{0}}^{\infty} \xi\left(\frac{1}{R(\xi)} \int_{\xi}^{\infty} Q(s) \mathrm{d} s\right)^{1 / \alpha} \mathrm{d} \xi=\int_{1}^{\infty} \xi \int_{\xi}^{\infty} \frac{\gamma}{s^{3}} \mathrm{~d} s \mathrm{~d} \xi=\frac{\gamma}{2} \int_{1}^{\infty} \xi^{-1} \mathrm{~d} \xi=\infty
$$

Denote the left hand side of (3.1) by $\psi\left(t_{*}\right)$. Then

$$
\begin{aligned}
\psi\left(t_{*}\right) & =\int_{t_{*}}^{\infty}\left(\frac{\gamma}{t^{2}} \frac{\int_{t_{2}}^{t / 3}\left(s-t_{1}\right) \mathrm{d} s}{\int_{t_{1}}^{t} \mathrm{~d} u}-\frac{9}{4 t}\right) \mathrm{d} t \\
& =\int_{t_{*}}^{\infty}\left[\frac{\gamma}{t^{2}\left(t-t_{1}\right)}\left(\frac{t^{2}}{18}-\frac{1}{2} t_{2}^{2}-\frac{1}{3} t_{1} t+t_{1} t_{2}\right)-\frac{9}{4 t}\right] \mathrm{d} t \\
& =\int_{t_{*}}^{\infty}\left(\frac{\gamma}{18\left(t-t_{1}\right)}-\frac{9}{4 t}-\frac{\gamma t_{1}}{3 t\left(t-t_{1}\right)}+\frac{\gamma\left(t_{1} t_{2}-0.5 t_{2}^{2}\right)}{t^{2}\left(t-t_{1}\right)}\right) \mathrm{d} t=\infty
\end{aligned}
$$


provided that $\gamma>40.5$. Hence, by Theorem 3.1, every solution $x$ of (4.1) is either oscillatory or satisfies $\lim _{t \rightarrow \infty} x(t)=0$ for any $\gamma>40.5$.

On the other hand, $\bar{Q}(t)=\min \left\{q\left(\sigma^{-1}(t)\right), q\left(\sigma^{-1}(\tau(t))\right)\right\}=\min \left\{\gamma /\left(27 t^{3}\right), 8 \gamma /\left(27 t^{3}\right)\right\}=$ $\gamma /\left(27 t^{3}\right)$, and so

$$
\liminf _{t \rightarrow \infty} \int_{\tau^{-1}(\sigma(t))}^{t} \frac{s^{2} \bar{Q}(s)}{r(s)} \mathrm{d} s=\liminf _{t \rightarrow \infty} \int_{\frac{2}{3} t}^{t} \frac{\gamma}{27 s} \mathrm{~d} s>\frac{5}{1.5 \mathrm{e}},
$$

provided that $\gamma>90 /(\mathrm{e} \ln 1.5) \approx 81.7$. An application of Theorem 1.1 implies that every solution $x$ of (4.1) is either oscillatory or satisfies $\lim _{t \rightarrow \infty} x(t)=0$ for all $\gamma>81.7$. Therefore, Theorem 3.1 improves Theorem 1.1. Observe that results reported in Baculíková and Džurina [1, 2], Candan [7], Džurina et al. [9], Jiang and Li [10], and Li et al. [14] cannot be applied to (4.1) due to the fact that $p(t)=2>1$.

Example 4.2 For $t \geq 1$, consider a third-order neutral delay differential equation

$$
\left((2+\sin t)\left[\left(x(t)+2 x\left(t-k_{1}\right)\right)^{\prime \prime}\right]^{3}\right)^{\prime}+\frac{\gamma}{t^{7}} x^{3}\left(t-k_{2}\right)=0,
$$

where $\gamma, k_{1}$, and $k_{2}$ are positive constants. Let $\alpha=3, k=1, r(t)=2+\sin t, p(t)=p_{0}=2$, $\tau(t)=t-k_{1}, \tau_{0}=1, q(t)=\gamma / t^{7}, f(u)=u^{3}, \sigma(t)=t-k_{2}, \rho(t)=t^{3}$, and $\delta(t)=0$. By virtue of $1 \leq r(t) \leq 3, \int_{1}^{\infty} r^{-1 / \alpha}(s) \mathrm{d} s=\infty$. It is not hard to see that assumptions $\left(A_{1}\right)-\left(A_{5}\right)$ hold. Noticing that $1 \leq R(t)=\max \{r(t), r(\tau(t))\}=\max \left\{2+\sin t, 2+\sin \left(t-k_{1}\right)\right\} \leq 3$ and $Q(t)=$ $\min \{q(t), q(\tau(t))\}=\min \left\{\gamma / t^{7}, \gamma /\left(t-k_{1}\right)^{7}\right\}=\gamma / t^{7}$, we obtain

$$
\begin{aligned}
\int_{t_{0}}^{\infty} \xi\left(\frac{1}{R(\xi)} \int_{\xi}^{\infty} Q(s) \mathrm{d} s\right)^{1 / \alpha} \mathrm{d} \xi & =\int_{1}^{\infty} \xi\left(\frac{1}{R(\xi)}\right)^{1 / 3}\left(\int_{\xi}^{\infty} \frac{\gamma}{s^{7}} \mathrm{~d} s\right)^{1 / 3} \mathrm{~d} \xi \\
& \geq\left(\frac{\gamma}{18}\right)^{1 / 3} \int_{1}^{\infty} \xi^{-1} \mathrm{~d} \xi \\
& =\infty
\end{aligned}
$$

Moreover,

$$
G(t)=\frac{t^{3}(2+\sin t)}{4^{4}}\left(\frac{3 t^{2}}{t^{3}}\right)^{4}+\frac{8 t^{3}\left(2+\sin \left(t-k_{1}\right)\right)}{4^{4}}\left(\frac{3 t^{2}}{t^{3}}\right)^{4} \leq \frac{3^{7}}{4^{4} t} .
$$

Denote the left hand side of (3.1) by $\psi\left(t_{*}\right)$. Then

$$
\begin{aligned}
\psi\left(t_{*}\right) & =\int_{t_{*}}^{\infty}\left[\frac{\gamma}{4 t^{4}}\left(\frac{\int_{t_{2}}^{t-k_{2}} \int_{t_{1}}^{s}(2+\sin u)^{-1 / 3} \mathrm{~d} u \mathrm{~d} s}{\int_{t_{1}}^{t}(2+\sin u)^{-1 / 3} \mathrm{~d} u}\right)^{3}-G(t)\right] \mathrm{d} t \\
& \geq \int_{t_{*}}^{\infty}\left[\frac{\gamma}{4 t^{4}}\left(\frac{\int_{t_{2}}^{t-k_{2}}\left(s-t_{1}\right) \mathrm{d} s}{3^{-1 / 3}\left(t-t_{1}\right)}\right)^{3}-\frac{3^{7}}{4^{4} t}\right] \mathrm{d} t \\
& =\int_{t_{*}}^{\infty}\left[\frac{\gamma}{4 t^{4}}\left(\frac{\left(t-k_{2}\right)^{2} / 2-t_{1} t+\beta}{3^{-1 / 3}\left(t-t_{1}\right)}\right)^{3}-\frac{3^{7}}{4^{4} t}\right] \mathrm{d} t \\
& =\infty
\end{aligned}
$$

provided that $\gamma>729 / 8=91.125$, where $\beta=-t_{2}^{2} / 2+t_{1} k_{2}+t_{1} t_{2}$. Therefore, by Theorem 3.1, every solution $x$ of (4.2) is either oscillatory or satisfies $\lim _{t \rightarrow \infty} x(t)=0$ for any $\gamma>91.125$. 
Theorem 1.1 and results of Baculíková and Džurina [4] do not allow us to arrive at this conclusion due to the fact that $\alpha \neq 1$. Observe that results reported in Baculíková and Džurina [1, 2], Candan [7], Džurina et al. [9], Jiang and Li [10], and Li et al. [14] cannot be applied to (4.2) since $p(t)=2>1$.

Remark 4.1 Without requiring assumptions (1.2) and (1.4), Theorem 3.1 is presented by using the double generalized Riccati substitutions (3.8) and (3.17). We stress that the study of oscillatory properties of equation (1.1) in the case $p(t)>1$ brings about additional difficulties. In particular, as in the papers by Baculíková and Džurina [4] and Li and Rogovchenko [12], we have to impose additional assumptions $\left(A_{5}\right)$. One of the principal difficulties one encounters lies in the fact that $x(t) \geq(1-p(t)) z(t)$ is not a valid estimate if $p(t)>1$ and $x$ is an eventually positive solution of (1.1). The question regarding the analysis of oscillatory behavior of solutions to (1.1) with other methods that do not require these assumptions remains open at the moment.

Remark 4.2 Note that Theorem 3.1 guarantees that every solution $x$ of (1.1) is either oscillatory or satisfies $\lim _{t \rightarrow \infty} x(t)=0$ and, unfortunately, this result cannot distinguish solutions with different behaviors. Since the sign of the derivative $z^{\prime}$ is not fixed, it is not easy to establish sufficient conditions which ensure that all solutions of (1.1) are just oscillatory and do not satisfy $\lim _{t \rightarrow \infty} x(t)=0$. Neither is it possible to use the technique exploited in this paper for proving that all solutions of (1.1) satisfy $\lim _{t \rightarrow \infty} x(t)=0$. Hence, these two interesting problems remain for future research.

Competing interests

The authors declare that they have no competing interests.

Authors' contributions

All three authors contributed equally to this work. They all read and approved the final version of the manuscript.

Author details

${ }^{1}$ Qingdao Technological University, Feixian, Shandong 273400, P.R. China. ${ }^{2}$ School of Informatics, Linyi University, Linyi, Shandong 276005, P.R. China. ${ }^{3}$ LinDa Institute of Shandong Provincial Key Laboratory of Network Based Intelligent Computing, Linyi University, Linyi, Shandong 276005, P.R. China.

\section{Acknowledgements}

The authors are grateful to the editors and three anonymous referees for a very thorough reading of the manuscript and for pointing out several inaccuracies. This research is supported by NNSF of P.R. China (Grant Nos. 61503171, 61403061, and 11447005), CPSF (Grant No. 2015M582091), NSF of Shandong Province (Grant No. ZR2012FL06), DSRF of Linyi University (Grant No. LYDX2015BS001), and the AMEP of Linyi University, P.R. China.

Received: 26 August 2015 Accepted: 16 June 2016 Published online: 30 June 2016

References

1. Baculíková, B, Džurina, J: Oscillation of third-order neutral differential equations. Math. Comput. Model. 52, 215-226 (2010)

2. Baculíková, $B$, Džurina, J: On the asymptotic behavior of a class of third order nonlinear neutral differential equations. Cent. Eur. J. Math. 8, 1091-1103 (2010)

3. Baculíková, B, Džurina, J: Oscillation theorems for second-order nonlinear neutral differential equations. Comput. Math. Appl. 62, 4472-4478 (2011)

4. Baculíková, B, Džurina, J: Oscillation theorems for higher order neutral differential equations. Appl. Math. Comput. 219, 3769-3778 (2012)

5. Baculíková, B, Džurina, J: Comparison theorems for higher-order neutral delay differential equations. J. Appl. Math. Comput. 49, 107-118 (2015)

6. Baculíková, B, Li, T, Džurina, J: Oscillation theorems for second-order superlinear neutral differential equations. Math Slovaca 63, 123-134 (2013)

7. Candan, T: Asymptotic properties of solutions of third-order nonlinear neutral dynamic equations. Adv. Differ. Equ. 2014, 35 (2014)

8. Candan, T: Oscillation criteria and asymptotic properties of solutions of third-order nonlinear neutral differential equations. Math. Methods Appl. Sci. 38, 1379-1392 (2015) 
9. Džurina, J, Thandapani, E, Tamilvanan, S: Oscillation of solutions to third-order half-linear neutral differential equations. Electron. J. Differ. Equ. 2012, 29 (2012)

10. Jiang, Y, Li, T: Asymptotic behavior of a third-order nonlinear neutral delay differential equation. J. Inequal. Appl. 2014 $512(2014)$

11. Li, T, Rogovchenko, YuV: Oscillation theorems for second-order nonlinear neutral delay differential equations. Abstr. Appl. Anal. 2014, 594190 (2014)

12. Li, T, Rogovchenko, YuV: Asymptotic behavior of higher-order quasilinear neutral differential equations. Abstr. Appl. Anal. 2014, 395368 (2014)

13. Li, T, Rogovchenko, YuV, Zhang, C: Oscillation results for second-order nonlinear neutral differential equations. Adv. Differ. Equ. 2013, 336 (2013)

14. Li, T, Zhang, C, Xing, G: Oscillation of third-order neutral delay differential equations. Abstr. Appl. Anal. 2012, 569201 (2012)

15. Şenel, MT, Utku, N: Oscillation criteria for third-order neutral dynamic equations with continuously distributed delay. Adv. Differ. Equ. 2014, 220 (2014)

16. Xing, G, Li, T, Zhang, C: Oscillation of higher-order quasi-linear neutral differential equations. Adv. Differ. Equ. 2011, 45 (2011)

Submit your manuscript to a SpringerOpen ${ }^{\odot}$ journal and benefit from:

- Convenient online submission

- Rigorous peer review

- Immediate publication on acceptance

- Open access: articles freely available online

- High visibility within the field

- Retaining the copyright to your article 